\title{
Responsabilidad social empresarial y segmentación de las cooperativas de ahorro y crédito, provincia de Loja- Ecuador ${ }^{1}$
}

\author{
Maritza Peña Vélez ${ }^{2}$ \\ maritza.pena@unl.edu.ec \\ https://orcid.org/0000-0002-9644-0822
}

Germania Sarmiento Castillo ${ }^{3}$

germania.sarmiento@unl.edu.ec https://orcid.org/0000-0002-6302-809X

Franklin Malla Alvarado ${ }^{4}$

franklin.malla@unl.edu.ec https://orcid.org/ 0000-0002-9170-3398

Filiación Universidad Nacional de Loja, carrera de Finanzas

Loja - Ecuador

\section{RESUMEN}

El objetivo de la investigación se relaciona con el análisis del cumplimiento de prácticas de responsabilidad social empresarial (RSE) en las cooperativas de ahorro y crédito (COAC) de la provincia de la Loja - Ecuador, segmentadas en base al volumen de sus activos de acuerdo con la normativa vigente. Se determina qué procesos se desarrollan en base a una caracterización de ochenta y dos actividades como indicadores de RSE en las treinta y dos cooperativas propias de cada cantón existente en la provincia, mediante información primaria obtenida por un cuestionario aplicado a sus directivos, en su mayoría gerentes. En la metodología diseñada para la investigación, se consideró el uso de la prueba Ji-Cuadrado, con un nivel de confiabilidad de 95\% para determinar si la dependencia es o no estadísticamente significativa, dimitiendo del resultado del que la H0 de cada indicador es válida, es decir que estas características estimadas como

\footnotetext{
${ }^{1}$ Artículo relacionado al proyecto original de investigación "Manual de Indicadores de responsabilidad social en las cooperativas de Ahorro y Crédito de la provincia de Loja”, U.N.L (2019 - 2021)

${ }^{2}$ Filiación Universidad Nacional de Loja, Carrera de Finanzas: Autor para correspondencia: maritza.pena@unl.edu.ec 3 Filiación Universidad Nacional de Loja, Carrera de Finanzas: Autor para correspondencia: gpilarsarmiento@gmail.com; germania.sarmiento@unl.edu.ec

${ }^{4}$ Filiación Universidad Nacional de Loja, Carrera de Finanzas: Autor para correspondencia: franklin.malla@unl.edu.ec
} 
indicadores de responsabilidad social son independientes del segmento al cual pertenece cada cooperativa, los resultados indicaron lo contrario en todas las dimensiones consideradas como actividades de RSE. Estos datos son valiosos y son el fundamento para la propuesta de indicadores de RSE dirigidos a las instituciones financieras pertenecientes a la Economía Popular y Solidaria.

Palabras clave: cooperativas; prácticas de RSE; dimensiones; caracterización 


\title{
Corporate social responsibility and segmentation of savings and credit cooperatives, Loja province - Ecuador
}

\begin{abstract}
The objective of the research is related to the analysis of compliance with practices of corporate social responsibility (CSR) in the savings and credit cooperatives (COAC) of the province of Loja - Ecuador, segmented based on the volume of their assets according to with current regulations. It is determined which processes are developed bases on a characterization of eighty-two activities as indicators of CSR in the province, through primary information obtained by a questionnaire applied to their managers, mostly managers in the methodology designed for the research, the use of the Chi-Square test was considered, with a confidence level of $95 \%$ to determine if the dependence is statistically significant or not, resigning from the result that the $\mathrm{HO}$ of each indicator is valid, in other words, these characteristics estimated as indicators of social responsibility are independent of the segment to which each cooperative belongs. The results indicated the opposite in all the dimensions considered as CSR activities. These data are valuable and are the basis for the proposal of CSR indicators aimed at financial institutions belonging to the Popular and Solidarity Economy.
\end{abstract}

Key Words: cooperatives; CSR practices; dimensions; characterization

Artículo recibido: 15 noviembre. 2021 Aceptado para publicación: 10 diciembre 2021 Correspondencia: maritza.pena@unl.edu.ec Conflictos de Interés: Ninguna que declarar 


\section{INTRODUCCIÓN}

El concepto de Responsabilidad Social Empresarial (RSE en adelante), es abordado desde diferentes perspectivas según estudios o proyectos que se llevan a cabo, no obstante, la relación con: medio ambiente, comunidad, sociedad, valores y principios éticos, marketing responsable, grupos de interés, son algunos de los conceptos que interactúan entre sí y modelan el proceso de RSE entendida como una visión o gestión empresarial que trabaja en forma sistémica en especial con indicadores que abarcan las tres dimensiones económica, social y medioambiental que se relacionan con los grupos de interés o llamados stakeholders, que en el caso de las cooperativas son sus propios socios o clientes, proveedores, y empleados.

La Comisión Europea (2011) en el mencionado Libro Verde, admite a la Responsabilidad Social Empresarial como "la integración voluntaria por parte de las empresas, de las preocupaciones sociales y ambientales en sus operaciones empresariales, y sus relaciones con sus interlocutores", es decir relaciona a los stakeholders con los indicadores de RSE que se menciona en este trabajo, además de tomar al Proyecto DERES (2001) desarrollado por la Universidad Católica de Uruguay como el referente a seguir en cuanto a su metodología de autoevaluación y medición de las prácticas de responsabilidad social empresarial al analizar los indicadores de:

Valores y principio éticos Condiciones de trabajo y empleo

$\longrightarrow$ Indicadores de la dimensión Social Apoyo a la comunidad

Marketing Responsable $\longrightarrow$ Indicadores de la dimensión Económica

Protección del medio ambiente $\longrightarrow$ Indicadores de la dimensión Ambiental DERES (2001) en el Manual de Autoevaluación de RSE, describe de manera clara y espontánea a cada uno de estos indicadores (p. 8)

Valores y principios éticos como el conjunto de medidas tomadas en cuenta en los procesos y objetivos estratégicos que se basan en ideales suscritos en los códigos de ética y conducta para la toma de decisiones organizacionales.

Condiciones de trabajo y empleo que hace referencia al manejo de los recursos humanos de recursos implementando prácticas como compensaciones, capacitación, ambiente 
laboral, promoción en su carrera administrativa, salud, seguridad laboral, y balance entre el trabajo, familia y tiempo libre.

Apoyo a la comunidad que se entiende como las múltiples acciones dirigidas hacia la comunidad que se relacionan con la oferta de servicios y productos, apoyo con recursos económicos, tiempo y capacitaciones, apoyo e incentivos a emprendimientos, todos aunados con la finalidad de obtener crecimiento económico y social de la comunidad.

Marketing Responsable que hace énfasis a las decisiones en los procesos legales y claros de divulgación de las características del producto o servicio que las empresas ofertan y que el consumidor debe conocerlas y recibirlas de la forma como las percibe.

Protección al medio ambiente manifestado como el compromiso de la empresa ante la conservación del medio ambiente y crecimiento sostenible de la organización integrando prácticas responsables para el efecto como el manejo de residuos contaminantes, capacitación y la concientización en el personal vinculado a la organización en temas de cuidado ambiental ya sea en las acciones dentro de la empresa o fuera de esta.

\section{MARCO TEÓRICO}

En los últimos 8 años las cooperativas de ahorro y crédito en el Ecuador se han caracterizado por consolidarse dentro del sector financiero popular y solidario como un baluarte de apoyo entre sus más de 6 millones 891 mil socios pertenecientes a 608 cooperativas activas a diciembre de 2019 según reporte de la Superintendencia de Economía Popular y Solidaria, convirtiéndose en una importante fuente de empleos formales en el país que trabajan para ejecutar los procesos financieros que impulsen a dicho sector y a otros de la economía de mercado.

En lo que respecta al desarrollo social, Orozco y Martínez (2017) indican que el eje fundamental de este son las empresas u organizaciones pertenecientes a todos los segmentos productivos, cumpliendo a cabalidad los objetivos tanto económicos como sociales y ambientales armonizando las relaciones con los stakeholders, precisamente sus empleados, alineando sus intereses con los de los demás grupos, es decir, proveedores y clientes; por ello las instituciones financieras (IF) al considerar a los empleados como un recurso productivo importante, deben proveer de las condiciones y ambiente laboral favorable para el desarrollo de las actividades, así como del espacio, equipo y mobiliario, ajustado a sus necesidades y políticas establecidas en un reglamento que ampare a cada uno de ellos. 


\section{Condiciones de trabajo y empleo}

La responsabilidad social empresarial (RSE), no es un acto altruista ni voluntario con miras a mejorar la imagen de la empresa, esta debe considerarse un proceso que permita mantener de forma sostenible principalmente a sus recursos humanos, es decir lograr la subsistencia económica y social relacionando los beneficios y utilidades para sus empleados, con el cuidado de la salud y prevención del aparecimiento de enfermedades laborales, asegurando de esta manera la permanencia de los colaboradores a largo plazo. Por otro lado, para cumplir el objetivo de obtener las ganancias en un sentido más amplio, se debe considerar la intervención de las organizaciones para combatir las enfermedades crónicas degenerativas de la profesión (Leiner de la Cabada, Ortíz y Ávila, 2008). También, se menciona al estrés laboral como una de las enfermedades de mayor impacto en los empleados, específicamente a los del sector financiero, bancos, cooperativas, etc., como una respuesta ante la demanda de obligaciones que se les imponga, provocando frustraciones cuando los resultados no son los esperados, y más aún, cuando la responsabilidad según el género es divida entre el trabajo y el hogar.

Abordando el tema de género en lo que respecta al empleado financiero estudios en Ecuador a nivel nacional como el de Mero, León y Guadalupe (2018), indican que al considerar a la mujer como dócil, débil y dependiente de la protección, la convierte en una persona con pocas habilidades de decisión, habilidad que la consideran nata en los hombres, consecuentemente, a estos últimos se les otorga y confía reponsabilidades y oportunidades en cargos gerenciales, como es el caso del establecimiento de esquemas eficientes de otorgamiento de crédito, lineamiento de los procesos de captaciones de valores legales y seguros, minimización de riesgos financieros entre otros. Los resultados superan la equidad de género con la balanza inclinada hacia el másculino, donde un $81 \%$ participan los hombres en contraste del $19 \%$ de cargos gerenciales y de directorio en las instituciones financieras del Ecuador ocupados por las mujeres.

En otro contexto dentro de la dimensión social en la RSC, el Manual de buenas prácticas para la inclusión laboral de personas con discapacidad (Ministerio de Relaciones Laborales, 2013), en su Art. 27. Trabajo y empleo, señala que

Los Estados Parte reconocen el derecho de las personas con discapacidad a trabajar, en igualdad de condiciones con las demás; ello incluye el derecho a tener la oportunidad de ganarse la vida 
mediante un trabajo libremente elegido o aceptado en un mercado y un entorno laborales que sean abiertos, inclusivos y accesibles a las personas con discapacidad (p.9).

Cabe destacar que se menciona además, la prohibición de la discriminación por la condición presentada, se asegura y permite a inclusión laboral, se alienta y promueve oportunidades a la par del establecimiento de programas de rehabilitación profesional. De esta manera y con la Ley que antecede, el Estado ecuatoriano se asegura que todas las empresas o instituciones se rijan a este marco legal so pena de sanciones y multas, cumplan con la inserción laboral de personas que actualmente son consideradas con capacidades diferentes más no discapacidad, logrando evitar el rechazo o miedo a incluirlas como parte de la población productiva sin que su condición afecte al normal desarrollo de los procedimientos en el ámbito laboral otorgándoles igualdad de oportunidades, y al superar los mínimos umbrales legales se estarían haciendo eco de la RSE.

Camacho (2015) considera la Responsabilidad Social como interna y externa; la primera aporta a la sociedad entregando productos y bienes de calidad, donde la eficiencia gerencial cumple un rol imprescindible en la selección del personal de la empresa, más aún, es importante la capacitación y formación continua ante un cúmulo y cambio de conocimientos, en especial técnicos y la creación de nuevas necesidades que deben ser satisfechas con la innovación de productos y servicios, y, es la Organización Internacional del Trabajo la que establece principios y derechos básicos que el Gobierno y directivos empresariales acogen y, ante la práctica de RSE en el país se han establecido programas como el Plan Nacional de Capacitación del Sector Público 2018 - 2021.

En el sector privado se hace imprescindible un proceso de capacitación continua que inicia con el análisis de las necesidades de recursos humanos que permitan adquirir las competencias para desempeñar el cargo, a decir de Ascencio \& Navarro (2015) los conocimientos de las personas son un activo intangible que no se ve reflejado en los balances contables de la empresa, pero si en la calidad de servicio, que, en el caso de las instituciones financieras, podrían mejorar la credibilidad y confianza atrayendo nuevos cuenta ahorristas y los valores depositados son aprovechados para la colocación de cartera de crédito y consiguiente incremento de la tasa de retorno de las COAC 
En la administración del talento humano además del proceso de capacitación, es imprescindible el manejo de rotación y retención del personal en sus puestos y actividades de trabajo, si bien es conocido que la rotación conlleva aspectos positivos para la empresa como eliminar la imprescindibilidad del empleado en casos ajenos a las decisiones erróneas de los directivos, también es indudable que estas prácticas traen consigo costos elevados, así lo indica el estudio de Mora et al (2017) donde señalan que la rotación de personal elevada es un peligro apremiante para la empresa, generando enormes costos a más de que el capital humano se desenvuelve en una batalla diaria generando ideas y produciendo bienes sin la estabilidad deseada, afectando de forma psicológica su productividad.

\section{Marketing responsable: calidad del servicio - investigación y desarrollo}

Al relacionar el marketing con la responsabilidad social empresarial, es importante destacar en primer lugar lo que significa un servicio, ya que es lo que ofertan las cooperativas de ahorro y crédito en todo su ámbito, por lo tanto, se lo considera como "un tipo de bien en el que predominan los componentes intangibles, frente al producto que sería aquel en el que predominan los componentes tangibles" (Arciniegas y Mejías, 2017) ${ }^{5}$; es decir un servicio se caracteriza principalmente por no ser percibido de manera visual pero que existe y cumple un fin específico para el cliente, de ahí que se debe analizar de manera oportuna la calidad del mismo con el fin de asegurar la permanencia del mismo y el nivel de resultados esperados.

Por otro lado "el marketing se ha integrado en la estrategia de las empresas hasta formar parte indisoluble de su ADN, como organizaciones que compiten en el mercado para ofrecer valor a sus clientes" (Andrade, 2016) ${ }^{6}$ es una medida que los administradores deben fortalecer continuamente sus medidas con el fin de que sus metas sean cumplidas a satisfacción de su inversionista, en la presente investigación los socios de las cooperativas. El marketing es una herramienta que permite anticiparse incluso a la competencia, toda vez que se da a conocer ciertos parámetros que la hacen única a la organización, pudiendo los clientes analizar ciertos beneficios que les permiten también maximizar sus capitales o a su vez contar con mayor seguridad de sus ahorros.

\footnotetext{
${ }^{5}$ En el estudio se aborda la percepción del cliente a través del modelo SERVQUALING el mismo que les permite conocer parámetros específicos de la organización y en función a ello plantear conclusiones tendientes a mejorar su situación actual.

${ }^{6} \mathrm{El}$ autor aborda el marketing digital como un aherramienta que les permite asegurarse su permanancia en el mercado, la aplicación eficas d ela misma determina el éxito de la unidad economica.
} 
Para llevar a cabo esta labor del marketing empresarial o corporativo, se requiere de profesionales idóneos en su área, ya que no sirve de mucho realizar campañas publicitarias o de conocimiento interno (percepción del cliente) si no se está cumpliendo con el fin específico para el cual fue creado el departamento. En esta parte las interrogantes planteadas a los socios deben estar eficazmente estructuradas, de manera tal que no importa la respuesta que obtengan, los administradores podrán conocer como ellos perciben el servicio ofertado por la cooperativa y en función a aquello mejorar sustancialmente su oferta en el corto plazo.

Ya adentrándose en el análisis sobre la satisfacción del cliente, estos estudios emergen a finales de los años 70 como una especialidad de investigación muy particular basadas en el conocimiento del comportamiento del consumidor (Nápoles, Tamayo y Moreno, 2016); esta situación también se relaciona con la realidad de que en los últimos años los clientes son más críticos con la calidad de los servicios que esperan de la entidad donde interactúan, siendo una tarea nada fácil pero que si se la aplica de forma oportuna, asegura el éxito de toda la organización.

Particularmente en las cooperativas de ahorro y crédito, el personal administrativo y operativo debe procurar que los socios lleven la mejor imagen de esta, ya que su criterio u opinión es compartida con otras personas del medio, que, en el futuro pueden convertirse en nuevos socios de la entidad, mejorando posiblemente el monto de captaciones que solventan los servicios financieros crediticios ofertados, finalmente, la situación influye en los resultados.

Por su parte González, Frías y Gómez (2016) señala que:

La calidad del servicio es una estrategia básica para enmarcar la estructura y la funcionalidad organizativa dentro de los parámetros que consideren las expectativas y necesidades de los clientes, la importancia del recurso humano para dar respuesta a estas exigencias debe ser determinantes para garantizar la calidad de los servicios que ofertan (p. 253).

Cuando una entidad financiera analiza la calidad de sus servicios a través de los mismos clientes (socios) puede considerarse una fortaleza, ya que conoce sus debilidades o lo que espera el cliente de la organización; su análisis y ejecución de acciones correctivas permite que el socio tenga una mejor apreciación de la institución y por tanto garantizar 
la continuidad de las operaciones con los recursos o capital necesario para su operatividad.

Para dar mayor soporte a la calidad de los servicios, la investigación y desarrollo juega un papel fundamental, ya que las innovaciones se producen a través de un proceso de aprendizaje continuo de las condiciones y acciones de la entidad y ente otras dependencias similares (Navas, 2017); esta interacción busca continuamente el perfeccionamiento de los servicios que se brinde con el fin de lograr la fidelidad del cliente (de donde salen los recursos para operar) y los resultados económicos planificados. En su análisis Loor y Carriel (2014) manifiesta que "la investigación y desarrollo (I+D) es un factor importante para el progreso de los países y el fomento de los sectores productivos" (p. 29); debido principalmente a que las entidades invierten flujos de efectivo para garantizar la mejora continua de sus ofertas, razón por la cual contratan, de no existir el capital humano disponible dentro de la empresa, a otras empresas dedicadas a esta labor a suministrar posibles alternativas de nuevas ofertas que permitan un crecimiento sostenible con la realidad de su entorno y sobre todo que cumplan el objetivo de servir a los socios y público en general en una problemática determinada. En el ámbito financiero de las cooperativas de ahorro y crédito esta inversión estaría destinada principalmente a mejorar las condiciones de financiamiento a los socios, a costos más favorables para las dos partes involucradas

\section{Protección del medio ambiente}

La responsabilidad social empresarial que asumen las organizaciones eficaces, también se asocia a la protección del medio ambiente, al respecto Rodríguez, Bustamante y Mirabal ( 2011) señalan que:

La falta de una cultura ambiental lamentablemente tiene un peso primordial en el surgimiento de los problemas que afectan al medio ambiente, y en las que se incluyen las indisciplinas, negligencias, indolencias y otras, que tanto tienen que ver con las costumbres y por ende con la moral ( $p, 517)$.

Esta premisa deja en claro que la responsabilidad social empresarial no debe estar alejada de los procedimientos o programas que asuman las entidades para proteger al medio ambiente, más aún, cuando son ellas las que por sus actividades crean desechos o emiten gases altamente dañinos para la naturaleza, de ahí que los directivos son los encargados 
de presupuestar fondos que estén encaminados a que sus residuos estén debidamente clasificados, reciclados o entregados a otras organizaciones para su tratamiento específico, minimizando el daño que se puede generar a su entorno natural.

Pérez, Espinoza y Peralta (2016) describen que una de las mejores formas de realizar responsabilidad social es precisamente a través del cuidado del medio ambiente, creando las estrategias pertinentes en el momento adecuado; por su parte Barba (2015) señala que este componente se asocia al análisis que se haga sobre los posibles impactos que una actividad económica pueda ejercer y sobre todo qué medidas se están ejecutando para prevenirlas. Finalmente, Marí (2016) detalla que la RSE medioambiental esta cada día más presente, en virtud de que existen ya políticas y leyes que la amparan, de ahí que existen mayores cuidados al momento de procesar sus desechos.

A manera de síntesis, el ser responsable con el medio ambiente no solo asegura el éxito de la empresa sino la vida mismo, de ahí que se debe buscar las alternativas necesarias para fortalecer su aplicación en todos los escenarios posibles y evitar catástrofes ya pronosticadas por importantes medios a nivel mundial.

\section{Apoyo a la Comunidad}

Navas y Londoño (2015) manifiestan que "la Responsabilidad Social Empresarial es percibida como generadora de beneficios, señalándose a la RSE como el compromiso que tiene la empresa de contribuir con el desarrollo, bienestar y el mejoramiento de la calidad de vida de los empleados, sus familias y la comunidad en general” (p. 89). Para Sanchez (2009) la gestión de responsabilidad social implica varias cosas, entre ellas, que las empresas desarrollen una visión de futuro con la comunidad y la sociedad en general; que se promueva liderazgos internos para la mejora de la productividad y la movilización de recursos humanos; la responsabilidad social es una estrategia de permanencia y legitimidad de la empresa, basada principalmente en que su fortaleza depende, en gran parte, del desarrollo social de la comunidad donde se establece.

Por su parte Saavedra garcía (2011) expresa que:

Las empresas que tienen un comportamiento socialmente responsable diseñan sus estrategias y establecen sus procedimientos internos de gestión teniendo en cuenta no sólo la dimensión económica de sus acciones, sino también la social y la medioambiental. Es decir, la RSE engloba todas las decisiones empresariales que son adoptadas por 
razones que a "primera vista" se encuentran más allá de los intereses económicos, financieros y técnicos de las empresas.

En este contexto, lo que la Responsabilidad Social establece que para que una empresa sea competitiva, no solo tiene que pensar en conseguir beneficios, sino que tiene que hacer compatible su labor productiva, con una política que actúe en beneficio de la comunidad en la cual se desarrolla; basándose en principios como el respeto a los derechos humanos, la mejora de las relaciones con la sociedad y el cuidado al medio ambiente. El servicio social de una empresa que lleva a cabo actividades de RSE, debe centrarse en: los empleados, comunidad, clientes y proveedores, derechos humanos, en la no discriminación, salud y seguridad en el trabajo, relaciones con sindicatos, entre otras.

Para Caravedo (1998) "La responsabilidad social es un compromiso que las empresas asumen por el bienestar del entorno social que las rodea" (p.15). Es por ello que ser una empresa con responsabilidad social, requiere de un modo de actuar honesto y respetuoso, lo cual se convierte en reconocimiento y aprecio por parte de sus clientes como un sello comprometido con la sociedad.

La responsabilidad social corporativa existe en la misma esencia de la entidad, está enmarcada en los valores y principios genuinos y esenciales del cooperativismo como doctrina, sistema y movimiento. No es un tipo especial de responsabilidad impuesta, ya que las cooperativas pueden actuar sin la mirada juiciosa del entorno porque han nacido de las mismas comunidades o son parte de ellas y no practican una forma articular de responsabilidad social empresarial, sino que son por y en sí mismas. (Gonzalez \& San Bartolome, 2008)

Sanchis y Rodríguez (2018) expresan que "Las cooperativas tienen una larga tradición al combinar viabilidad económica y responsabilidad social gracias al diálogo entre las partes interesadas y a la gestión participativa, y pueden servir de referencia a otras" (p. 219).

La responsabilidad social para el sector cooperativo financiero se puede definir al conjunto de actividades que son el resultado de su voluntad por servir a los diversos sectores sociales, se puede desarrollar mediante programas que permitan mejorar la calidad de vida de los clientes o socios y comunidad en general, enfatizando en cada uno de ellos aspectos como la no discriminación, el respeto a los derechos humanos, la 
aceptación a la diversidad, entre otros, buscando alcanzar la consolidación y participación comprometida de socios y empleados en busca de mejores condiciones de vida.

\section{Valores y Principios Éticos}

Una empresa es socialmente responsable cuando promueve el desarrollo de prácticas transparentes a lo interno y externo de la empresa, es por ello que al hablar de responsabilidad social empresarial es preciso hacer referencia al desempeño ético de las organizaciones.

León (2017) manifiesta que "la ética de la organización se relaciona con las características individuales y que estas categorías están asociadas directamente con el comportamiento no ético: a) constructos de personalidad, b) valores de orientación y c) otras características individuales" (p. 164). Por su parte Begoña y Martínez (2015) expresan que:

Una empresa es socialmente responsable cuando su modelo de actuación (su buen gobierno) promueve el desarrollo de prácticas transparentes en el foro interno y en el externo de la empresa. Este marco de actuación no se limita solo al área de confort de la organización o a los resquicios que no se recogen en las leyes, sino a un saber hacer ético (p. 39).

La Responsabilidad Social debe estar basada en principios y valores que regulen las relaciones y la conducta de todos miembros de la organización, la satisfacción de los clientes no acaba con la calidad de los productos o los servicios, hoy los grupos sociales están demandando nuevos bienes y servicios que incorporan cuestiones éticas en su producción (equidad, respeto con el medio ambiente y justicia social); han hecho que los riesgos sociales y medioambientales sean hoy más significativos que en el pasado.

Luquez y Mejía (2012) señalan que la responsabilidad social y la ética, "representan una ventaja competitiva para las organizaciones hoy en día, en donde estas pueden llegar a evolucionar hasta llegar a conciliar los intereses de los accionistas con las demandas de los diferentes grupos de intereses con los que interactúa" (p. 97).

En este contexto García (2000) "manifiesta que la responsabilidad social es un recurso estratégico fundamentado en la responsabilidad moral" (p. 50). Con ello se puede determinar que la ética empresarial prácticamente es obligatoria para asegurar el funcionamiento correcto de las organizaciones, es decir se debe asumir un modelo de 
actuación basado en principios éticos que se debe ver reflejado en todas las acciones de la empresa.

Carrasco (2005) manifiesta que:

Una empresa socialmente responsable también crea valor cuando ofrece un buen servicio al cliente, a precios competitivos $y$ proporcionándole bienes y servicios de calidad; de igual modo, también crea valor cuando paga un precio justo, sin abuso de posiciones de dominio de mercado a los proveedores; o cuando remunera a los asalariados de forma justa, preocupándose por su estabilidad laboral y su formación (p. 353).

Por lo tanto, la responsabilidad social empresarial integra en ella el respeto por los valores éticos que inciden en las personas, las comunidades y el medio ambiente. Belhouari, Buendía, Lapointe, y Tremblay (2005) manifiestan que "en el sector financiero, las entidades tienen cada vez mayor interés en las cuestiones éticas como reflejo de un compromiso empresarial para satisfacer la demanda de la sociedad en general" (p. 192), de esta forma funcionan de una manera eficiente a medio plazo, evitando la competencia desleal que pudiera generar la no adopción de prácticas de orientación social.

Finalmente se puede concluir que las prácticas de Responsabilidad Social al interior del sector financiero están enfocadas en una serie de valores, actitudes y comportamientos que definen la actuación de las empresas y afectan a los actores corporativos (trabajadores o colaboradores); así mismo las prácticas externas están vinculadas a la totalidad de la cadena productiva (proveedores, consumidores y clientes) y dentro de ello abarcan asuntos ambientales y sociales.

\section{Relación entre Responsabilidad Social Empresarial RSE y empresas}

En este apartado se describen aportes significativos de la relación existente entre la RSE y las empresas, donde también interactúan las cooperativas de ahorro y crédito, factores muy importantes para entender la dimensión de su aplicación y los beneficios que puede conllevar su aplicación de manera oportuna.

En su investigación Espitia (2015) señala lo siguiente:

La RSE no es sólo es un tema de moda, sino que se constituye en una tendencia que cada vez adquiere mayor relevancia, ya que su cumplimiento permite que el papel de las mipymes en la sociedad (como 
entes constructores, transformadores y desarrolladores de esta) se cumpla con ética empresarial, mejorando la calidad de vida de los miembros de cada organización, sus familias y su entorno (p. 82).

Es decir, con la implementación de los parámetros de la RSE, las organizaciones tienden a ser más solidarias con sus integrantes y a su vez con su entorno, esta realidad se ve fortalecida si se relaciona con el sector cooperativo financiero de Ecuador, que tiene una participación importante en la actividad de canalizar créditos a los sectores más vulnerables en todas sus regiones. La ética en la ejecución de acciones de RSE es clave para que los resultados se los pueda alcanzar y cristalizar en beneficio del talento humano de la empresa que lo elabora, así como de la sociedad en general. (Belhouari, Buendía Martínez, Lapointe, \& Tremblay, 2005)

Por su parte Villacís, Suarez y Güillín (2016) menciona que:

Las normas y estándares internacionales de RSE sirven como guía y ejemplo para las empresas que buscan formar parte de los negocios responsables y sostenibles, así como también para los organismos reguladores que buscan promover una industria con consciencia social. Constituyen el marco para realizar auto crítica a sus prácticas empresariales y para plantear decisiones y proyectos de mejora en cada ámbito analizado. El cumplimiento de la Responsabilidad Social no se hace por exigencia o imposición, sino por voluntad propia, por ética o consciencia individual y empresarial y como una forma de correspondencia de las empresas con la sociedad en la que opera, en la que se desarrolla y para la cual trabaja (p.463).

En esta acápite, es necesario mencionar que en Ecuador existen muchos incentivos tributarios para varias empresas, uno de ellos es el sector cooperativo en toda su dimensión, ya que las empresas pertenecientes a este grupo económico, por poner un ejemplo no están obligados al pago del impuesto a la renta si pueden demostrar que sus excedentes han sido reutilizados en sus operaciones normales; es ahí justamente donde nace la necesidad de que exista un mayor compromiso en el cumplimiento de a RSE incluso como estrategia de competitividad, ya que las acciones que se puedan ejercer para mejorar el medio ambiente, condiciones laborales, apoyo a fundaciones sociales entre otros son bien percibidos por la población en general. 
Cabe recalcar que el cumplimiento de prácticas de RSE aún no es obligatorio en el país, pero pese a aquello son varias cooperativas de ahora y crédito que ya vienen realizando esfuerzos para darles cumplimiento y son precisamente aquellas organizaciones que han logrado reconocimiento a nivel nacional e internacional. Adicionalmente, con la globalización, las entidades buscan un posicionamiento a nivel internacional, siendo la RSE una oportunidad para lograrlo, ya que existen organizaciones que reconocen la labor emprendida desde sus planificaciones.

En su análisis Aguilera (2012) hace énfasis a la relación entre la RSE y el crecimiento económico sobre la base de alternativas estratégicas que se las resume de la siguiente forma:

- Beneficios para los grupos de interés.- cuando las empresas generan responsabilidad empresarial, esta situación se puede convertir en incremento de utilidades, ampliación de operaciones, apoyo a la capacitación del personal, generación de nuevas plazas de empleo, mayor inversión social (jordanas de salud, mejoramiento de parques, entre otros); es necesario recordar que la RSE permite un crecimiento empresarial y a través del crecimiento empresarial se obtienen recursos para mejorar o ampliar programas de RSE.

- La reputación de la empresa. - para iniciar es necesario entender que la reputación empresarial es el resultado de un proceso competitivo mediante el cual las empresas transmiten sus características, maximizando su estatus moral y socioeconómico. En este sentido, al mostrar una gestión ejemplar, manteniendo resultados estables, cumpliendo con las obligaciones tributarias y laborales, la entidad logra que su reputación se fortalezca a través de los años, todo ello con prácticas sólidas de RSE en todas sus dimensiones, lo que se revierte en una mayor demanda de los productos o servicios que se oferten en el mercado.

- Las acciones socialmente responsables. - es muy importante destacar que los problemas de la sociedad pueden ser oportunidades de negocio; es decir, conviene actuar éticamente, ya que con ello se redunda en beneficios para la entidad a corto o largo plazo; realizar obras de gran impacto social, permite un mayor posicionamiento en las mentes de los grupos de interés. 
- La honestidad y justicia. - cuando la empresa ha cometido errores y estos son asumidos con responsabilidad, puede ocasionar ganancias de reputación a la compañía, ya que siempre será positivo que la empresa tenga deseos sinceros de remediar sus equivocaciones.

- Crecimiento económico sostenible. - las organizaciones adquieren cada día mayor conciencia que permita un desarrollo sostenible con el medio ambiente, garantizando las fuentes de recursos naturales y contribuyendo a su conservación.

- Las tecnologías de la información y comunicación TIC. - el avance den las TIC está presente en todos los ámbitos de la economía; relacionándolo con la RSE, los clientes prefieren comprar en empresas que persigan una conducta ética y asumen compromisos y retos de RSE.

- La producción limpia. - las empresas deben buscar la producción limpia, estableciendo políticas medioambientales en sus compras, minimizando sus residuos contaminantes y sobre todo estableciendo un sistema óptimo de reciclaje, aprovechando al máximo las materias primas y por ende la reducción de los costos.

- La responsabilidad social interna. - el crecimiento de la empresa cuando utiliza de manera eficaz la RSE se ve reflejada en el fortalecimiento de la confianza y el compromiso de sus empleados, se ve más motivada y se crea una cultura organizacional que fomente la generación de valor en cada operación que se ejecute. Finalmente, Duque, Cardona y Rendón (2013) en relacion al aporte de todos los sectores sociales en fomentar la RSE, señala que:

Ya es un acto de responsabilidad con la sociedad que desde la academia y las empresas se dé una oposición a las pretensiones de hacer de la RSE un concepto de moda: no en vano, el concepto ha venido fortaleciéndose a lo largo de las últimas décadas y debe ser menester de las instituciones públicas y privadas lograr acuerdos nacionales e internacionales para posibilitar la unificación de los parámetros de medición y lograr de esta manera hacer que la RSE se convierta en un concepto potente de gestión empresarial, con ética y compromiso social, político, cultural, ambiental y económico (p.205). 
Esta premisa facilita entender que la RSE no solo debe ser asumida como un simple concepto o definición actual, sino que su implementación debe estar acompañada de una planificación acorde a la realidad de cada organización social o económica y en función de mejorar sustancialmente la calidad de vida de todos en general, priorizando al ser humano y la naturaleza que pueden estar siendo vulnerados por las acciones que se emprenden desde el giro normal de sus operaciones. Más allá de conseguir las metas de rentabilidad esperadas por los inversionistas, las acciones encaminadas a dejar una sociedad mejor son las que permiten que la unidad económica o empresa sea catalogada como responsable y tengan la aceptación favorable de toda la sociedad.

\section{MATERIALES Y MÉTODOS}

Para el desarrollo de este estudio donde se aplicó la herramienta cuestionario de prácticas de RSC efectuadas en el año 2020, cuyas interrogantes adoptan el modelo validado DERES (2001) se acogerá a un enfoque correlacional o de asociatividad, aplicando la prueba estadística Chi cuadrado con un nivel de confianza del 95\%, la que permite abordar sobre la relación entre la caracterización de las actividades como indicadores dentro de las dimensiones de la Responsabilidad Social Empresarial (RSE) y el tamaño de las cooperativas (medido según la segmentación de activos) en la actualidad.

Se trabajó con el total o universo de datos al ser factible la aplicación del cuestionario a cada cooperativa autóctona de cada cantón, es decir donde opera la matriz.

El estudio se centra en la comprobación de la significancia en la asociatividad entre las variables descritas, ya que indudablemente si se deseara determinar la causalidad y al ser esta un juicio de valor, se requiere más información o variables explicativas que junto a la RSE, determinen por ejemplo la intervención de estas en el crecimiento de las COAC. Los datos que intervienen en el estudio de las cooperativas pertenecientes a cada cantón de la provincia de Loja y clasificadas por la Superintendencia de Economía Popular y Solidaria por segmento de acuerdo con el valor de sus activos, se presenta en la siguiente tabla: 
Tabla 1.

Cooperativas locales activas de la provincia de Loja

\begin{tabular}{|c|c|c|c|}
\hline $\begin{array}{l}\text { Nombre de las cooperativas según } \\
\text { segmentos }\end{array}$ & $\begin{array}{l}\text { Número de } \\
\text { Cooperativas }\end{array}$ & $\begin{array}{c}\text { Activos } \\
\text { (USD) }\end{array}$ & $\begin{array}{c}\% \\
\text { Segmento }\end{array}$ \\
\hline $\begin{array}{l}\text { Cooperativas } \\
\text { (Cooperativa de ahorro y crédito } \\
\text { COOPMEGO). }\end{array}$ & 1 & $\begin{array}{c}\text { Mayor a } \\
80.000 .000,00\end{array}$ & $3 \%$ \\
\hline $\begin{array}{l}\text { Cooperativas Segmento 2: (Coop. } \\
\text { Padre Julián Lorente, CACPE, } \\
\text { CACEL). }\end{array}$ & 3 & $\begin{array}{c}\text { Mayor a } \\
\text { 20.000.000,00 } \\
\text { hasta } \\
80.000 .000,00\end{array}$ & $9 \%$ \\
\hline $\begin{array}{l}\text { Cooperativas Segmento 3: (Cristo } \\
\text { Rey, CACPE Célica, Coop. } \\
\text { FORTUNA, Crediamigo). }\end{array}$ & 4 & $\begin{array}{c}\text { Mayor a } \\
5.000 .000,00 \\
\text { hasta } \\
20.000 .000,00\end{array}$ & $13 \%$ \\
\hline $\begin{array}{l}\text { Cooperativas Segmento 4: (Coop. } \\
\text { Saraguros, Semillas del Progreso, } \\
\text { Coop del Comerciante, CADECOM, } \\
\text { Quilanga, Gonzanamá, CADECOG, } \\
\text { Catamayo, CACVIL, Obras Públicas } \\
\text { Fiscales de Loja y Zamora, Servidores } \\
\text { Municipales de Loja, Solidaria). }\end{array}$ & 12 & $\begin{array}{c}\text { Mayor a } \\
1.000 .000 .00 \\
\text { hasta } \\
5.000 .000,00\end{array}$ & $38 \%$ \\
\hline $\begin{array}{l}\text { Cooperativas Segmento 5: (Coop. } \\
\text { ECOSUR, Inticoop, Las Lagunas, } \\
\text { Santiago, } 23 \text { de Enero, COPYMEC, } \\
\text { Cariamanga, } 22 \text { de Junio, San José } \\
\text { Airo, "Instituto Daniel Álvarez } \\
\text { Burneo", CACDESUR, San } \\
\text { Sebastián). }\end{array}$ & 12 & $\begin{array}{c}\text { Hasta } \\
1.000 .000,00 \\
\text { Cajas de Ahorro, } \\
\text { Bancos } \\
\text { Comunales y } \\
\text { Cajas Comunales }\end{array}$ & $38 \%$ \\
\hline TOTALES & 32 & & $100 \%$ \\
\hline
\end{tabular}

Nota: adaptado de estadísticas SEPS

Los datos son procesados en IBM SPSS Statistics V25.0 tomando las variables segmentos e indicadores de RSE para analizar las hipótesis planteadas a un nivel de significancia de 0.05. Los indicadores en totalidad cuentan con 82 ítems relacionados a la RSE como parámetros de análisis de las 32 COAC, resultando en un total de 2624 respuestas válidas. 


\section{RESULTADOS Y DISCUSIÓN}

En el estudio descriptivo, los resultados se muestran en el porcentaje de cumplimiento de los indicadores de responsabilidad social empresarial de acuerdo con cada uno de los segmentos.

\section{Tabla 2}

Cumplimiento de los indicadores considerados como actividades de RSE

\begin{tabular}{|c|c|c|c|c|c|c|c|c|c|c|c|}
\hline \multirow{2}{*}{$\begin{array}{c}\text { Seg. } \\
1\end{array}$} & & \multicolumn{2}{|c|}{$\begin{array}{c}\text { NO } \\
\text { CORRESPONDE }\end{array}$} & \multicolumn{2}{|c|}{ NUNCA } & \multicolumn{2}{|c|}{ A VECES } & \multicolumn{2}{|c|}{ SIEMPRE } & \multirow{2}{*}{$\begin{array}{c}\text { TOTAL } \\
25\end{array}$} & \multirow{2}{*}{$\begin{array}{c}\% \\
100 \%\end{array}$} \\
\hline & & 0 & $0.00 \%$ & 0 & $0.00 \%$ & 0 & $0.00 \%$ & 25 & $100.00 \%$ & & \\
\hline 2 & & 2 & $2.67 \%$ & 2 & $2.67 \%$ & 6 & $8.00 \%$ & 65 & $86.67 \%$ & 75 & $100 \%$ \\
\hline 3 & $\begin{array}{l}\text { Trabajo y } \\
\text { Empleo }\end{array}$ & 3 & $3.00 \%$ & 24 & $24.00 \%$ & 21 & $21.00 \%$ & 52 & $52.00 \%$ & 100 & $100 \%$ \\
\hline 4 & & 17 & $5.67 \%$ & 52 & $17.33 \%$ & 56 & $18.67 \%$ & 175 & $58.33 \%$ & 300 & $100 \%$ \\
\hline 5 & & 10 & $3.33 \%$ & 83 & $27.67 \%$ & 66 & $22.00 \%$ & 141 & $47.00 \%$ & 300 & $100 \%$ \\
\hline 1 & & 0 & $0.00 \%$ & 0 & $0.00 \%$ & 0 & $0.00 \%$ & 13 & $100.00 \%$ & 13 & $100 \%$ \\
\hline 2 & & 2 & $5.13 \%$ & 0 & $0.00 \%$ & 0 & $0.00 \%$ & 37 & $94.87 \%$ & 39 & $100 \%$ \\
\hline 3 & Marketing & 0 & $0.00 \%$ & 12 & $23.08 \%$ & 13 & $25.00 \%$ & 27 & $51.92 \%$ & 52 & $100 \%$ \\
\hline 4 & & 4 & $2.56 \%$ & 8 & $5.13 \%$ & 21 & $13.46 \%$ & 123 & $78.85 \%$ & 156 & $100 \%$ \\
\hline 5 & & 2 & $1.28 \%$ & 13 & $8.33 \%$ & 35 & $22.44 \%$ & 106 & $67.95 \%$ & 156 & $100 \%$ \\
\hline 1 & & 0 & $0.00 \%$ & 0 & $0.00 \%$ & 0 & $0.00 \%$ & 10 & $100.00 \%$ & 10 & $100 \%$ \\
\hline 2 & & 4 & $13.33 \%$ & 2 & $6.67 \%$ & 10 & $33.33 \%$ & 14 & $46.67 \%$ & 30 & $100 \%$ \\
\hline 3 & $\begin{array}{c}\text { Medio } \\
\text { Ambiente }\end{array}$ & 3 & $7.50 \%$ & 20 & $50.00 \%$ & 11 & $27.50 \%$ & 6 & $15.00 \%$ & 40 & $100 \%$ \\
\hline 4 & & 20 & $16.67 \%$ & 48 & $40.00 \%$ & 20 & $16.67 \%$ & 32 & $26.67 \%$ & 120 & $100 \%$ \\
\hline 5 & & 16 & $13.33 \%$ & 53 & $44.17 \%$ & 20 & $16.67 \%$ & 31 & $25.83 \%$ & 120 & $100 \%$ \\
\hline 1 & & 0 & $0.00 \%$ & 0 & $0.00 \%$ & 0 & $0.00 \%$ & 14 & $100.00 \%$ & 14 & $100 \%$ \\
\hline 2 & & 6 & $14.29 \%$ & 0 & $0.00 \%$ & 7 & $16.67 \%$ & 29 & $69.05 \%$ & 42 & $100 \%$ \\
\hline 3 & $\begin{array}{l}\text { Apoyo a la } \\
\text { Comunidad }\end{array}$ & 5 & $8.93 \%$ & 23 & $41.07 \%$ & 8 & $14.29 \%$ & 20 & $35.71 \%$ & 56 & $100 \%$ \\
\hline 4 & & 5 & $2.98 \%$ & 28 & $16.67 \%$ & 35 & $20.83 \%$ & 100 & $59.52 \%$ & 168 & $100 \%$ \\
\hline 5 & & 9 & $5.36 \%$ & 45 & $26.79 \%$ & 59 & $35.12 \%$ & 55 & $32.74 \%$ & 168 & $100 \%$ \\
\hline 1 & & 0 & $0.00 \%$ & 0 & $0.00 \%$ & 0 & $0.00 \%$ & 20 & $100.00 \%$ & 20 & $100 \%$ \\
\hline 2 & $\begin{array}{l}\text { Valores y } \\
\text { Principios }\end{array}$ & 0 & $0.00 \%$ & 0 & $0.00 \%$ & 1 & $1.67 \%$ & 59 & $98.33 \%$ & 60 & $100 \%$ \\
\hline 3 & Éticos & 0 & $0.00 \%$ & 9 & $11.25 \%$ & 16 & $20.00 \%$ & 55 & $68.75 \%$ & 80 & $100 \%$ \\
\hline 4 & & 9 & $3.75 \%$ & 12 & $5.00 \%$ & 30 & $12.50 \%$ & 189 & $78.75 \%$ & 240 & $100 \%$ \\
\hline 5 & & 16 & $6.67 \%$ & 25 & $10.42 \%$ & 35 & $14.58 \%$ & 164 & $68.33 \%$ & 240 & $100 \%$ \\
\hline & Total respue & as vá & & & & & & & & 2624 & \\
\hline
\end{tabular}


Todos los indicadores de RSE en el segmento 1 obtienen un 100\% de cumplimiento ya que estos se refieren a los datos de la participación de solamente una cooperativa de ahorro y crédito perteneciente a dicho segmento, por ello los siguientes análisis hacen referencia a las COAC de los segmentos dos y tres, que poseen un capital entre 5 y 80 millones USD totalizando siete cooperativas y con un capital menor a 5 millones USD resultando en 24 cooperativas de la provincia de Loja de los segmentos cuatro y cinco. El indicador trabajo y empleo que hace referencia al manejo responsable y solidario de los recursos humanos dentro de cada cooperativa, el segmento 2 cumple un $86,67 \%$ en condición de "siempre" en la caracterización de todos los factores que están asociados a este indicador, seguido por el cumplimiento de siempre por los segmentos 4, 3 y 5 en ese orden. Por otro lado, el segmento 5 es el que obtiene mayor \% entre todos al responder en un $27,67 \%$ que el manejo de recursos humanos no lo hacen con tendencia a prácticas responsables y solidarias.

En lo que se refiere a la variable Markerting que hace énfasis a las decisiones de divulgación de las características del producto o servicio que las empresas ofertan y entregan al consumidor, el segmento 2 obtiene el 94,87\% de cumplimiento total en todos los ítems, y al igual que el indicador trabajo y empleo, es seguido por los segmentos 4, 3 y 5 en ese orden de cumplimiento. El segmento 3 es el que mayor porcentaje obtiene al contestar un $23.08 \%$ que, en algunas características referentes a la promoción de productos y entrega, no lo han hecho con base a la responsabilidad social.

El estudio del indicador Medio Ambiente guía al compromiso de la empresa ante la conservación y crecimiento sostenible; integrando prácticas responsables para el efecto; el segmento 1 obtiene el 100\% de respuestas en condición "siempre" en la caracterización de todos los factores que están asociados, pero es necesario indicar que los segmentos 2 , 3 y 4 y 5 cumplen menos del $50 \%$ en lo que se refiere a realizar buenas prácticas dirigidas a la conservación del medio ambiente.

En relación con el indicador apoyo a la comunidad, el segmento 2 satisfactoriamente realiza prácticas donde involucra a los stakeholders como socios, proveedores y comunidad en general en el $69,05 \%$ de las oportunidades que son requeridas, el segmento 4 lo hace en el 59.52\% seguidos por las participaciones menores de los segmentos 3 y 5 . Finalmente, las prácticas de RSE basadas en los códigos de ética y conducta, se indica que todos los segmentos del sector cooperativista financiero las desarrollan en, el $98.33 \%$ 
(segmento 2); 68.75\% (segmento 3); $78.75 \%$ (segmento 4) y, 68.33\% (segmento 5), siendo este indicador el que mayor proporción entre las cooperativas ha alcanzado en relación a los 5 indicadores propuestos dentro de las dimensiones de RSE.

\section{Planteamiento y contraste de hipótesis}

Se plantean las hipótesis de investigación $H_{0}$ de independencia y las consiguientes hipótesis alternativas para cada indicador como prácticas de RSE.

$H_{0 a}$ : Las prácticas relacionadas al trabajo y empleo son independientes del segmento cooperativo

$H_{1 a}$ : Las prácticas relacionadas al trabajo y empleo son dependientes del segmento cooperativo

$H_{0 b}$ : El marketing responsable es independiente del segmento cooperativo

$H_{1 b}$ : El marketing responsable es dependiente del segmento cooperativo

$H_{0 c}$ : Las prácticas medioambientales son independientes del segmento cooperativo

$H_{1 c}$ : Las prácticas medioambientales son dependientes del segmento cooperativo

$H_{0 d}$ : El apoyo a la comunidad es independiente del segmento cooperativo

$H_{1 d}:$ El apoyo a la comunidad es dependiente del segmento cooperativo

$H_{0 e}$ : Los valores y principios son independientes del segmento cooperativo

$H_{1 e}$ : Los valores y principios son dependientes del segmento cooperativo

En la tabla se muestran los valores obtenidos por cada indicador respecto al segmento, bajo para prueba de chi cuadrado.

Tabla 3

Pruebas de asociatividad - chi-Cuadrado

\begin{tabular}{lllcccc}
\multicolumn{1}{c}{ Indicadores } & $\begin{array}{l}\text { Chi-cuadrado } \\
\text { de Pearson }\end{array}$ & Valor & df $\begin{array}{c}\text { Significación } \\
\text { asintótica } \\
\text { (bilateral) }\end{array}$ & $\begin{array}{c}\text { N de } \\
\text { casos } \\
\text { válidos }\end{array}$ & $H_{0}$ \\
\hline a.) Trabajo y Empleo & $\begin{array}{l}\text { Chi-cuadrado de } \\
\text { Pearson }\end{array}$ & $67,482^{\mathrm{a}}$ & 12 & 0,000 & 800 & Se Rechaza H0a \\
\hline b.) Marketing & $\begin{array}{l}\text { Chi-cuadrado de } \\
\text { Pearson }\end{array}$ & $47,932^{\mathrm{a}}$ & 12 & 0,000 & 416 & Se Rechaza H0b \\
\hline c.) Medio Ambiente & $\begin{array}{l}\text { Chi-cuadrado de } \\
\text { Pearson }\end{array}$ & $48,771^{\mathrm{a}}$ & 12 & 0,000 & 320 & Se Rechaza H0c \\
\hline $\begin{array}{l}\text { d.) Apoyo a la } \\
\text { comunidad }\end{array}$ & $\begin{array}{l}\text { Chi-cuadrado de } \\
\text { Pearson }\end{array}$ & $77,328^{\mathrm{a}}$ & 12 & 0,000 & 448 & Se Rechaza H0d \\
\hline e.) Valores y principios & $\begin{array}{l}\text { Chi-cuadrado de } \\
\text { Pearson }\end{array}$ & $43,916^{\mathrm{a}}$ & 12 & 0,000 & 640 & Se Rechaza H0e \\
\hline
\end{tabular}


Al realizar las pruebas estadísticas para determinar la correlación y dependencia entre las características evaluadas como indicadores de responsabilidad social empresarial de las COAC y el segmento al cual pertenecen con un nivel de significancia del 5\%, grados de libertad=12, conociendo los valores de chi-cuadrado para los indicadores, estos resultaron mayores en todos los casos al indicativo en la tabla, así mismo como significación asintótica en las cinco pruebas resultó $\mathrm{p}<0,05$, consecuentemente, las hipótesis planteadas $H_{0}$ se rechazan en todos los casos y se aceptan las hipótesis alternativas $H_{1}$, donde se determina que cada indicador dentro de las dimensiones de RSE ejecutadas por cada cooperativa, son dependientes del segmento al cual pertenecen. Los resultados obtenidos analizan la dependencia de las acciones de RSE de cada cooperativa, es decir sus actividades como instituciones responsables y solidarias que bien pueden ser utilizadas para incrementar la credibilidad de los socios y futuros socios en sus procesos y demanda de servicios, es así que cotejando con estudios como el de Iturrioz del Campo, Masa, y Medina ( 2019) que señalan que el tamaño de las empresas del sector financiero de España pone en manifiesto la predisposición de publicar datos sobre desarrollo de RSE justificacndo su comportamiento en general. En lo que se refiere a las acciones puntuales como las relacionadas a Gobierno Corporativo, son informadas por todas las empresas sin distinción de tamaño y más aún las acciones sociales que influyen en la liquidez que estas presentan; en lo que respecta a los resultados de rentabilidad económica y financiera, concluyen que estos no son afectados significativamente por la promoción de las acciones de RSE que desarrolla, mas, no señalan que el ejecutarlas no implique efecto en la rentabilidad.

Finalmente, consecuencia de diferentes motivaciones, como es el caso del incremento en la credibilidad de los clientes, se afirma que estos confiarán indudablemente en el trabajo que realiza la institución financiera entregando sus ahorros que son fondos utilizados para la colocación en futuros créditos e incrementar el número de operaciones financieras que repercutan en sus excedentes, y, por otro lado, mediante el estadístico utilizado en la investigación, se establece que el tamaño (medido por la segmentación) de la cooperativa es el que determina que actividades de RSE se ejecutan, además de que existe dependencia estadísticamente significativa. El grupo de investigación ha considerado importante esta conclusión, siendo necesario que, para comprobar la opción de que las prácticas de RSE son las que influyen en el crecimiento empresarial, se debe contar con 
los registros de presupuestos anuales de algunos periodos contables asignados al desarrollo de las actividades que incluyan indicadores de responsabilidad social.

\section{CONCLUSIONES}

Los valores resultantes determinan en el presente caso que cada cooperativa ejecuta procesos de RSE definidos por su tamaño, situación que conlleva a que se establezca en la propuesta de la metodología de indicadores para las COAC, un sistema que tome en cuenta esta particularidad. Los procesos están expresados porcentualmente, esto permite que las cooperativas puedan relacionar sus prácticas con procesos de RSE de otras instituciones financieras que en esta investigación resultaron significativamente dependientes de su tamaño.

Al proponer en el futuro un modelo de indicadores de RSE dirigidos exclusivamente a las cooperativas de ahorro y crédito pertenecientes al sector popular y solidario mas no dentro del sistema financiero del Ecuador que contempla a los bancos, sociedades financieras, (controlados por la Superintendencia de Bancos) y, cualquier otro distinto de las COAC (controladas por la Superintendencia de Economía Popular y Solidaria, SEPS), se debe identificar las áreas que requieran un tratamiento especial y mayor atención para que mejoren su desempeño en lo posterior, como por ejemplo a la población que no tiene acceso a la inclusión financiera y que no es considerada por los bancos como posibles clientes.

Los aspectos que quedan abiertos a debate para estudios futuros, es establecer la implicación de la RSE dentro de las instituciones financieras de ahorro y crédito como un determinante de sus excedentes, es decir si existe o no tal escenario, por ello la necesidad e importancia de que los organismos encargados de receptar la información financiera de estas cooperativas publiquen los datos en el portal de la SEPS, en la actualidad esta es incompleta, requiriéndose información o "banco de datos" de todos los segmentos y así inferir los resultados en conclusiones que explican la injerencia de cada proceso ya sea administrativo, financiero etc., relacionado a las prácticas de RSE y estas a su vez en qué medida influyen en el crecimiento de las cooperativas.

\section{REFERENCIAS BIBLIOGRÁFICAS}

Aguilera Castro, A. (2012). Crecimiento empresarial basado en la Responsabilidad Social. Revista científica Pensamiento y Gestión, 1-15. Obtenido de 
http://rcientificas.uninorte.edu.co/index.php/pensamiento/article/view/3977/386 $\underline{4}$

Andrade Yejas, D. A. (2016). Estrategias de marketing digital en la promoción de Marca Ciudad. Revista Escuela de Administración de Negocios, 59 - 72. Obtenido de http://www.redalyc.org/articulo.oa?id=20645903005

Arciniegas Ortiz, J. A., \& Mejías Acosta, A. A. (2017). PERCEPCIÓN DE LA CALIDAD DE LOS SERVICIOS PRESTADOS POR LA UNIVERSIDAD MILITAR NUEVA GRANADA CON BASE EN LA ESCALA SERVQUALING, CON ANÁLISIS FACTORIAL Y ANÁLISIS DE REGRESIÓN MÚLTIPLE. COMUNI@CCION, 26-36. Obtenido de http://www.redalyc.org/articulo.oa?id=449852109003

Ascencio, E., \& Navarro, J. (2015). Importancia de la capacitación y el desarrollo del talento humano en el Ecuador. Observatorio de la economía latinoamericanca, (207).

Barba Bayas, D. (2015). Responsabilidad Social Empresarial: Estudio de Casos en Riobamba. Revista Ciencia UNEMI, 50-58.

Begoña Gómez, N., \& Martínez Domínguez, R. (2015). Los valores éticos en la responsabilidad social corporativa. ANAGRAMAS, 38. Obtenido de http://www.scielo.org.co/pdf/angr/v14n28/v14n28a02.pdf

Belhouari, A., Buendía Martínez, I., Lapointe, M. J., \& Tremblay, B. (2005). La responsabilidad social de las empresas: ¿un nuevo valor para las cooperativas? CIRIEC-España, Revista de Economía, 191-208. Obtenido de https://www.redalyc.org/pdf/174/17405313.pdf

Camacho, J. (2015). Las normas de responsabiidad social. Su dimensión en el ámbito laboral de las empreas. Revista latinoamericana de derecho social, (20), 3-29.

Caravedo, B. (1998). Responsabilidad social de la empresa: un eje para cambiar el país. Fundación Ambiente y Recursos Naturales, 1-63.

Carrasco, I. (2005). La ética como eficiencia: la responsabilidad social en las cooperativas de ahorro y crédito españolas. CIRIEC-España, Revista de Economía Pública, Social y Cooperativa, 351-367. Obtenido de https://www.redalyc.org/pdf/174/17405320.pdf 
DERES, O. S. (07 de Septiembre de 2001). https://www.academia.edu/10710428/EMPRESARIAL_Otras_publicaciones_de _DERES_Manual_de_Primeros_Pasos_en_RSE_Manual_de_Autoevaluaci\%C3 $\% B 3 n \_d e \_R S E \_M a n u a l \_p a r a \_l a \_P r e p a r a c i \% C 3 \% B 3 n \_e \_I m p l e m e n t a c i \% C 3 \%$ B3n_del_.

Duque Orozco, Y. V., Cardona Acevedo, M., \& Rendón Acevedo, J. A. (2013). Responsabilidad Social Empresarial: Teorías, índices, estándares y certificaciones. Cuadernos de Administración, 196-206. Obtenido de https://www.redalyc.org/pdf/2250/225029797009.pdf

Espitia Cubillos, A. A. (2015). La responsabilidad social empresarial (RSE) como elemento estratégico en las mipymes colombianas. Ingeniería Industrial. Actualidad y Nuevas Tendencias, 75-84. Obtenido de https://www.redalyc.org/pdf/2150/215047422008.pdf

García Marza, D. (2000). Responsabilidad Social y Balance Social de la Empresa. Madrid- Fundación Mapfre.

González Arias, M., Frías Jiménez, R. A., \& Gómez Figueroa, O. (2016). Análisis de la calidad percibida por el cliente en la actividad hotelera. Ingeniería Industrial, 253265. Obtenido de http://scielo.sld.cu/scielo.php?script=sci_arttext\&pid=S1815$\underline{59362016000300004}$

Gonzalez, L., \& San Bartolome, J. (2008). Balance Social Cooperativo una construcción en construcción. Obtenido de http://www.aciamericas.coop/IMG/pdf/lgonzalez2.pdf

Iturrioz del Campo, J., Masa, C., \& Medina, A. (18 de Julio de 2019). La transparencia sobre Responsabilidad Social Corporativa y su implicación con el comportamiento económico -financiero: efectos en las empresas de Economía Social. (E. Complutense, Ed.) Revista de Estudios Cooperativos, Vol.132, p. 22, (122-143).

Leiner de la Cabada, M., Ortíz, J., \& Ávila, C. (2008). Un nuevo paradigma de la responsabilidad social corporativa: el aumento de enfermedades crónicodegenerativas en la fuerza laboral. Contaduría y administración, (225), 105 - 119. 
León Saltos, A. (2017). Los valores éticos y la responsabilidad social corporativa en las Cooperativas de Ahorro y Crédito de la ciudad de Ambato. Ciencia y Tecnología al servicio del pueblo, 160-170.

Loor, M. F., \& Carriel, V. (2014). Investigación y Desarrollo en Ecuador: un análisis comparativo entre América latina y el Caribe (2000-2012). COMPENDIUM, 2846.

Luquez Rangel, O., \& Mejía Alvarado, M. (2012). Ética como enfoque de la responsabilidad social: direccionamiento estratégico en universidades públicas. Red de revistas científicas de América Latina, el Caribe, España y Portugal, 95108. Obtenido de https://www.redalyc.org/pdf/737/73723402007.pdf

Marí Farinós, J. (2016). LA RESPONSABILIDAD SOCIAL MEDIOAMBIENTAL. ANÁLISIS JURÍDICO DE LA DIRECTIVA 2004/35/CE SOBRE RESPONSABILIDAD MEDIOAMBIENTAL*: QUIEN CONTAMINA PAGA. DIFERENCIAS CON LA RESPONSABILIDAD SOCIAL EMPRESARIAL (RSE). Revista Boliviana de Derecho, 254-273. Obtenido de http://www.redalyc.org/articulo.oa?id=427543196012

Mero, M., León, J., \& Guadalupe, R. (2018). Las polítias del Buen vivir: una mirada a la equidad de género laboral en el sector financiero ecuatoriano. Espacios, Vol. 39 (No.28) Pág. 25.

Ministerio de Relaciones Laborales (MRL). (s.f.). Manual de buenas prácticas para la inclusión laboral de personas con discapacidad. Obtenido de Ministerio del Trabajo: http://www.trabajo.gob.ec/wpcontent/uploads/downloads/2013/12/MANUALFIN.pdf

Ministerio de Relaciones Laborales. (Diciembre de 2018). Trabajo.gob.ec. Obtenido de http://www.trabajo.gob.ec/wp-content/uploads/2015/11/Plan-Nacional-deCapacitaci\%C3\%B3n-2018.pdf

Mora, J., Antonieta, J., Huilcapi, M., \& Paredes, J. (2017). Gestión empresarial frente a la rotación del personal. Observatorio de Economía latinoamericana, http://www.eumed.net/cursecon/ecolat/ec/2017/gestion-empresarialecuador.html.

Nápoles-Nápoles, L. Y., Tamayo-García, P., \& Moreno-Pino, M. (2016). Medición y mejora de la satisfacción del cliente interno en instituciones universitarias. 
Ciencias Holguín, 1-16. Obtenido de http://www.redalyc.org/articulo.oa?id=181545579003

Navas Olmedo, W. H. (2017). Investigación e innovación, factores de crecimiento en las PYMES. Revista Publicando, 254-268.

Navas Ríos, M. E., \& Londoño Aldana, E. (2015). LAS FUNDACIONES Y SU CONCEPCIÓN Y GESTIÓN DE LA RESPONSABILIDAD SOCIAL. Saber, Ciencia $y \quad$ Libertad, $87-100 . \quad$ Obtenido de: https://revistas.unilibre.edu.co/index.php/saber/article/view/873

Orozco, Y., \& Martínez, D. (2017). Responsabilidad social empresarial en la dimensión laboral: caso Bancolombia y BBVA. Revista Facultad de Ciencias Económicas, 20(1), 171-187.

Pérez Espinoza, M. J., Espinoza Carrión, C., \& Peralta Mocha, B. (2016). La responsabilidad social empresarial y su enfoque ambiental:una visión sosteniblea futuro. Universidad y Sociedad, 169-178.

Rodríguez Morales, V., Bustamante Alfonso, L. M., \& Mirabal Jean-Claude, M. (2011). La protección del medio ambiente y la salud, un desafío social y ético actual. Revista Cubana de Salud Pública, 510-518. Obtenido de http://www.redalyc.org/articulo.oa?id=21421364015

Saavedra garcía, M. L. (2011). La Responsabilidad Social Empresarial y las Finanzas. Cuadernos de Administración, 39-54. Obtenido de http://www.scielo.org.co/pdf/cuadm/v27n46/v27n46a04.pdf

Sanchez, M. d. (2009). Responsabilidad Social. Una mirada desde la Psicología Comunitaria. Liberabit. Revista de Psicología, 163-170. Obtenido de https://www.redalyc.org/pdf/686/68611924010.pdf

Sanchis Palacio, J. R., \& Rodríguez Pérez, S. (2018). Responsabilidad social empresarial en banca. Su aplicación al caso de la banca cooperativa. REVESCO. Revista de Estudios Cooperativos, 204-227. Obtenido de https://pdfs.semanticscholar.org/e1ba/49aef65a7cff1afb30ef773189d339bf304d. pdf

Superintendencia de Economía Popular y Solidaria. (2018). Rendición de cuentas del Sector Económico Popular y Solidario. Quito. 
Responsabilidad social empresarial y...

Villacís Laínez, C. A., Suarez Núñez, Y. F., \& Güillín Llanos, X. M. (2016). Análisis de la Responsabilidad Social en el Ecuador. Revista Publicando, 452-466. Obtenido de : https://revistapublicando.org/revista/index.php/crv/article/view/295 